\title{
Peril Elements of Infection Severity, Disease Non-Improvement, Case Fatality and Management of Sars-Cov-2 in Sargodha Region Pakistan
}

\author{
Amar Naziri', Nazia Nazir ${ }^{2}$, Muhammad Mustafa Qamar ${ }^{3}$, Asifa Alia ${ }^{4}$, Fida Muhammad Sheikh ${ }^{5}$, Amna Mubeen ${ }^{6}$ \\ 1 Associate Professor, Department of Medicine, Sargodha Medical College, Sargodha Pakistan \\ 1 Main idea, Concept and writing \\ 2 Consultant Psychiatrist. Govt. General Hospital G.M Abad Faisalabad Pakistan \\ 2 Proof reading \\ 3 Assistant Professor, AHS. Sargodha Medical College, Sargodha Pakistan \\ Discussion \\ 4 Assistant Professor, Department of Gynecology, Rai Medical College, Sargodha Pakistan \\ 4 Literature Review \\ 5 Assistant Professor, Department of Cardiology, Sargodha Medical College, Sargodha Pakistan \\ 5 Introduction \\ 6 Assistant Professor, Department of Anatomy, Sargodha Medical College, Sargodha Pakistan \\ 6 References

 \\ of Sars-Cov-2 In Sargodha Region Pakistan. APMC 2021;15(1):86-90. DOI: 10.29054/APMC/2021.1012
}

\section{ABSTRACT}

Background: Corona virus is +ve stranded RNA virus pervasive in most of the atmospheres and towards the end of year 2019 it originated a pandemic as emerging microorganism which started in Wuhan city of China causing mild respiratory illness to severe acute hypoxemic respiratory distress syndrome in humans causing lot of deaths. Thanks to Almighty ALLAH that its virulence has been decreased in recent past in Pakistan due to better government planning and preventive measures. Objective: To manage moderate to severe COVID- 19 patients with pneumonias in HDU/ attached wards with central oxygen supply and to observe peril elements of infection severity, non-improvement and case fatality. Study Design: Descriptive, cross sectional, multicenter and heterogenous study of fifty COVID positive patients. Settings: High Dependency Unit \& Isolation (Pulmonology ward) District Head Quarter Hospital Sargodha \& Niazi Teaching Hospital (OPD), Sargodha Pakistan. Duration: From 15 May 2020 to 30 June 2020. Methodology: Fifty COVID- 19 patients over eighteen years of age presented with moderate to severe illness were enrolled for management in this study. Patients having +ve RT- PCR test (nasopharyngeal swab) for COVID-19/ CT chest with pneumonias, pyrexia, cough, tachypnoea with or without confusion, coma, dehydration, fits, low oxygen saturation, feeding difficulties, myocardial or renal injuries, raised liver enzymes, dysfunctional coagulation, expeditious disease advancement with respiratory failure (ARDS) were included in this study for management. Results: Every patient was admitted for moderate to severe COVID 19 pneumonias, tachypnoea etc. Eleven patients needed high flow nasal oxygen (HFNO) humidified oxygen or noninvasive ventilation (NIV) and non-rebreather mask and two patients required invasive ventilation, 24 patients (48\%) patients had diabetes mellitus with complications, 8 patients $(16 \%)$ had chronic obstructive airway disease (COAD) or asthma on steroids and hypertension with complications was diagnosed in $6(12 \%)$ patients as well. As a whole two critically ill (one old aged diabetic) patients died over one and a half month of study period. Conclusion: COVID-19 can be a fatal disease especially in patients with old age, chronic illnesses (diabetes, COAD, hypertension and chronic renal failure etc.), immunocompromised states, early detection with management is of great value. This study interprets that early usage of NIV (CPAP and HFNO) diminishes respiratory failure symptoms, worsening of disease and the need for invasive ventilation.

Keywords: Moderate to severe COVOD-19 patients Peril elements of disease severity NIV and mechanical ventilation.

\section{INTRODUCTION}

COVID -19 is a recent person to person transferable human viral illness, its disease course and acrimony are at a halt even not fully understandable and treatment may progress as pandemic continues. ${ }^{1}$ In Pakistan first case of lethal corona virus was affirmed on February 2020 up till which it was declared as worldwide pandemic. ${ }^{2}$ Major routes of spread are contact with infected persons, droplets of cough, sneezing and this virus can enter in humans through nose, mouth and eyes. Corona virus can infect at a distance of about six feet, it can survive on surfaces for few hours to even days and touching these surfaces may also be the infection source. ${ }^{3}$ SARS- COV-2 (severe acute respiratory syndrome) is a novel corona virus and was first detected at Wuhan city China. In January 19, 2020 first reported cases were in the state of 
Washington and since then more than 7000000 cases of COVID -19 have been reported with about 0.3 million deaths since then. ${ }^{4}$ This illness has spread in more than 215 countries worldwide and still without any specific treatment. ${ }^{5}$ In our Sargodha city cases continued to rise towards mid of June 2020 but after that there is gradual decline of this awful disease by grace of Almighty Allah. Better Governmental planning (lock downs), low humidity and high temperatures may also be the contributing factors for its low transmission rates.

Under electron microscope corona virus surface has various spikes helping it to bind and attack human living cells. Corona virus is usually of $20 \mathrm{~nm}$ in size having positive single stranded RNA genome with symmetrical helical nucleocapsid and appearance of surface is club or large petal shaped. Bats and some other animals are usual source of this virus and it belongs to coronaviridae family. 6,7 Slow mutations are important features of this virus causing problems in its treatments, control and its vaccination. Raised counts of cytokines, chemokines, Creactive proteins, leukocytes and other pro- inflammatory cells are usual associated pathological conditions of corona virus. Its common symptoms can be mild respiratory symptoms as dry cough, sore throat, sneezing with pyrexia of high grade, body aches, fatigue and can be severe hypoxemic respiratory failure, pneumonias, cardiac and renal involvements and death even. 8,9

50 Patients included in this study were confirmed PCR (nasopharyngeal swab) or radiologically (CXR and CT chest) proven COVID- 19 with moderate to severe illness who were admitted in HDU of DHQ Hospital Sargodha from 15 May to 30 June 2020. In this study the utmost try was to save patient's lives so no drugs comparisons were done (as still there is no specific treatment option for this illness). Immunocompromised patients as diabetics, with malignancies, chronic lung, renal or cardiac diseases and old aged people are at increased risk of this infection. Staying at home, regular washing of hands with soapssanitizers, cleaning your home- office areas, things of regular use, use of masks, washing foods before cooking and avoidance of public transport, public places, contact with persons having flu like symptoms are usual preventive measures of this disease. ${ }^{10,11}$

\section{METHODOLOGY}

Study Design: Descriptive cross-sectional study.

Settings: High Dependency Unit \& Isolation (Pulmonology ward) District Head Quarter Hospital Sargodha \& Niazi Teaching Hospital (OPD), Sargodha Pakistan.

Duration: From 15 May 2020 to 30 June 2020.

Sample Size: Fifty COVID positive patients

Data Collection Procedure: Patients having +ve RT- PCR test for COVID-19/ CT chest with pneumonias (lung infiltrates less than $50 \%$ of lung fields), pyrexia, shortness of breath with low oxygen saturation less than $94 \%$, cough, fatigue, headache, myalgias, (moderate disease) and with confusion, coma, dehydration, fits, low oxygen saturation with respiratory rate more than 30 breaths/ minute, feeding difficulties, myocardial or renal injuries, raised liver enzymes, dysfunctional coagulation, expeditious disease advancement with respiratory failure (ARDS) having widespread infiltrates on immediate CXR (lung infiltrates more than $50 \%$ of lung fields), septic shock, CURB 3 or 4 score (severe disease), requiring constant ICU monitoring, invasive ventilation, NIV through CPAP or HFNO were included in this study. Seriously ill patient's assessment was done with hourly vitals, neurological monitoring, constant pulse oximetry with additional observation of accessory muscles usage, decline in breathing pattern, tachypnoea, decreased oxygen saturations ( $\mathrm{PaO} 2$ less than $80 \mathrm{mmHg}$ ) or increased oxygen demands and periodic ABGs. Acute respiratory distress syndrome (ARDS) was explained as sudden onset hypoxemia with $\mathrm{P} / \mathrm{F}$ ratio (arterial $\mathrm{PO} 2$ from $\mathrm{ABG} / \mathrm{FIO} 2$ that is percent of inspired $\mathrm{O} 2$ by patient) $<300$ with chest $X$ ray infiltrates with no evidence of congestive cardiac failure or volume overload.

As Contemporary information from different analysis recommended no particular treatment for human COVID- 19 infection but best available treatment based on recent available information was started in moderate to severely ill patients. NIV was done after patients consent with age more than $18, \mathrm{SpO} 2$ less than $92 \%$ in spite of usual low oxygen flow therapy of $6 \mathrm{~L} / \mathrm{min}$ for at least15-20 min. Patients needing direct invasive ventilation, pneumothorax, unconsciousness and PCO2 more than $6 \mathrm{kpa}$ were excluded from NIV.

NIV (in isolation) as high Flow Nasal cannula oxygen (HFNC) (20-70L/min) oxygen, non- rebreather mask (NRB), frequent prone positioning, Bilevel Positive Airway Pressure (BiPAP in COVID- 19 with COPD), Continuous Positive Airway Pressure (CPAP with observation on side effects like barotraumas and pneumothorax) were used in severely ill patients associated with complex respiratory conditions with 3045 degrees of bed elevation.

Under strict protective guidelines and least number of staff invasive mechanical ventilation was performed in patients having ARDS with severe respiratory failure having poor oxygen reserves (consistent $\mathrm{SpO} 2$ less than 90\% despite CPAP with $\mathrm{FiO} 2$ more than 0.6-0.7). These patients were also refractory to CPAP/NIV with agitation or confusion and worsening respiratory acidosis. Early targeted neuromuscular blockage, deep sedation, head elevation of bed, diminished fluid administration and buildup were done in patients with severe ARDS or having mechanical ventilation. Tidal volumes were restricted to $4-8 \mathrm{ml} / \mathrm{kg}$ of body weight for pulmonary protective ventilator scenarios and with respiratory rate of 12-15breaths/ min. High flow oxygen was needed for 2 patients who received mechanical ventilation shortly 
after its initiation with plateau pressures to less than 30 $\mathrm{cm} \mathrm{H} 2 \mathrm{O}$ (24-26) and driving pressure of about $14-16 \mathrm{~cm}$ $\mathrm{H} 2 \mathrm{O}$ or as needed. Most favorable measures of PEEP (approximately $5 \mathrm{~cm} \mathrm{H} 2 \mathrm{O}$ ) and element of permissive hypercapnia was also there.

All critically ill patients and intubated ARDS patients were nursed with careful fluids management, diuretics especially in patients of congestive cardiac failure, enoxaparin (LMWH) or rivaroxaban according to Ddimmers value, combination of broad-spectrum antibiotics, corticosteroids (inj. dexamethasone $6 \mathrm{mg}$ od), zinc plus vitamin D, awake proning 12-14 hours, tocilizumab $8 \mathrm{mg} / \mathrm{kg}$ IV once or twice according to criteria and for fever acetaminophen were used for these patients treatment. Patients discharge criteria was for patients having symptoms ten days after start of features plus three extra days with no respiratory symptoms or fever and one day apart two consecutive -ve PCR tests and if this patient is still +ve after five days a repeat sample should be obtained.

\begin{tabular}{|l|c|}
\hline HDU management procedures & No of patients out of 50 \\
\hline CPAP & 7 \\
\hline HFNC & 14 \\
\hline Invasive Ventilation & 2 \\
\hline Prone positioning & 35 \\
\hline Vasopressors & 14 \\
\hline Neuromuscular blockade & 2 \\
\hline Inhaled bronchodilators & 30 \\
\hline Outcomes & \\
\hline Mortality & 2 \\
\hline
\end{tabular}

Statistical Analysis: Data illustrated in percentage, and mean. Data was analyzed using Statistical package for social sciences (SPSS) software for windows version 23.

\section{RESULTS}

50 patients (39 men and 11 women) with affirmed Covid19 were included in this study. Out of these fifty patients forty-one patients had moderate and 9 patients had severe illness. The mean age of these patients was $48.76 \pm$ 13 years (Figure 1 ).

About all patients had history of shortness of breath and cough, $60 \%$ had expectoration, $65 \%$ patients had fever (all features $5 \pm 2$ days). Every patient was admitted for moderate to severe COVID- 19 pneumonia. The sign and symptoms of the patients of COVID-19 are presented in table 1. Majority of the patients presented with Diabetes millets (Table 1).
Figure 1: Percentage of HDU management procedures

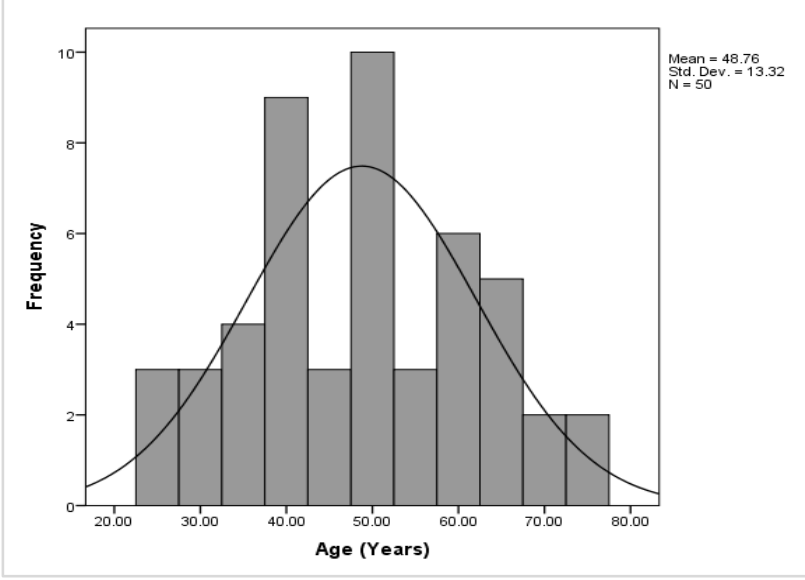

Table 1: Sign and symptoms of patients presented with COVID-19

\begin{tabular}{|c|c|c|c|}
\hline \multicolumn{2}{|c|}{ Symptoms on presentation } & \multicolumn{2}{|c|}{ Signs on presentation } \\
\hline $\begin{array}{l}\text { Shortness of breath } \\
\text { and cough }\end{array}$ & $94 \%$ & $\begin{array}{l}\text { Respiratory Rate } \\
\text { more than } 25\end{array}$ & $88 \%$ \\
\hline Expectoration & $60 \%$ & $\begin{array}{l}\text { Pulse rate more than } \\
\qquad 100\end{array}$ & $80 \%$ \\
\hline Fever & $65 \%$ & Fever more than 101 & $70 \%$ \\
\hline Sore throat & $26 \%$ & & \\
\hline Rhinorrhea & $22 \%$ & & \\
\hline Headache & $16 \%$ & & \\
\hline
\end{tabular}

All the patients advised for chest $X$ rays and 22 patients asked for CT scan, all serious patients needed NIV or HFNO and two patients required invasive mechanical ventilation. The NIV required for 11 days on average and invasive ventilation duration was 7 days. Fourteen patients were hypotensive and treated by norepinephrine, dopamine and other inotropes. As a whole 2 patients died over one and a half month of study period. This study projected that noninvasive ventilation and high flow nasal cannula oxygen are befitting measures for managing progressive pneumonias due to COVID and the patients can be escaped from the requirement of invasive ventilation. In this study NIV or mechanical ventilation of patients were done in HDU (separated in cabins) of DHQ Hospital Sargodha under constant nursing and doctors supervision. In our study many patients had long standing illnesses before HDU admission as 24 patients (48\%) patients had diabetes mellitus with complications like nephropathy, IHD etc., 8 patients $(16 \%)$ had COAD with +ve smoking history (via MDI with spacer bronchodilators delivered) or asthma on steroids and most of the patients also had lymphocytopenia on presentation and hypertension with complications was diagnosed in $6(12 \%)$ patients as well. 
Figure 2: Peril elements

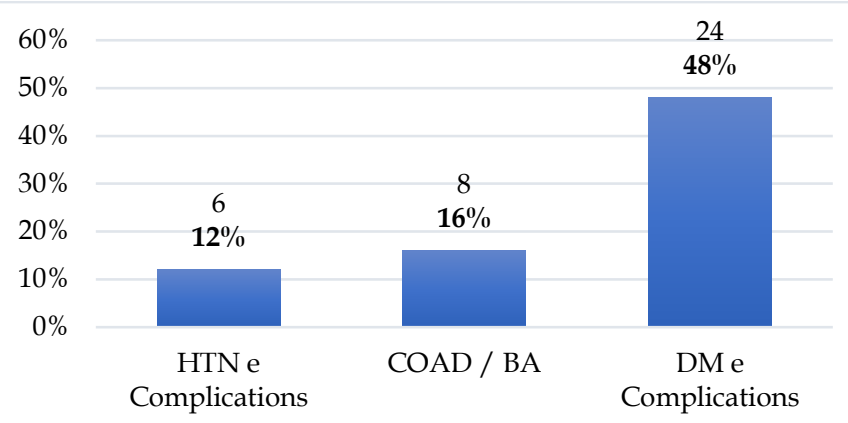

- Percentage $\%$

\section{DISCUSSION}

This infectious disease (COVID- 19 pandemic) has dreadful effects on human's health so It's necessary to adhere strictly with quarantine rules, all preventive and management measures to combat its hazardous consequences and complications. ${ }^{12}$ Our study is multicenter series of cases which narrates fifty moderates to seriously ill patients who were admitted due to infection of confirmed COVID-19 (laboratory/ radiologically proved) with moderate to severe respiratory failure and hypoxemia during the period of 15th May to 30th June 2020. Sputum and blood cultures were also sent to laboratories. SARS- COV-2 presenting symptoms were about similar in patients as described from China, Italy and USA which shows a customary host behavior. Fever was relatively less common symptom as compare to shortness of breath and cough in these COVID- 19 patients on admission to high dependency unit DHQ Sargodha so noting pyrexia only can hold up diagnosis so other mentioned symptoms are of utmost importance for early diagnosis as well.

In our study. Some seriously ill patients also had raised white blood counts and neutrophils due to underlying secondary bacterial infection. Eight patients had unbalanced renal function tests, five patients had deranged liver function tests, many patients had raised $\mathrm{LDH}$, ferritin and CRP levels and there were bilateral pneumonias on chest imaging of all patients.

- The case casualty rate of only $4 \%$ in this study is comparable with one study by J. Zhang et al in which mortality rate was $3.77 \%$ including moderate to severely ill patients of COVID- 19.13 Protracted proinflammatory response of patients with inadequate control of viral replication causes immune dysfunction leading poor outcomes in patients especially in elderly and our study suggests that old age persons were more prone to infections with poor outcomes and unimprovement. ${ }^{14}$ In our moderately severe COVID- 19 patients mortality rate is $4 \%$ which is less than revealed in earlier studies but surpassing in another previously reported study.
Differences on the basis of case incorporation criterion probably is the reason for this discrepancy. ${ }^{15,16}$ Other reasons can be deficient early guidelines of management, no specific treatment, a different race, difference in number of patients and our study includes most of the patients with moderate illness.

- Nevertheless, according to our results mortality rates are closer to the official national stats which is 3.34\%. Some patients presented with shock and lack of bacterial (streptococcus, staphylococcus) co infections suggested that it's directly relevant to Covid-19. These patients were treated with dopamine or norepinephrine etc. and echocardiogram was normal in most of them. Four patients of bronchial asthma also presented with moderately severe Covid-19 respiratory failure and two of them required mechanical ventilation. Glucocorticoids were started in severely ill patients as studies on COVID- 19 acute respiratory distress syndrome proposed ameliorated outcomes in these patients. ${ }^{17,18}$

\section{CONCLUSION}

Diabetes mellitus with long standing complications especially nephropathy and ischemic heart disease, chronic obstructive airway disease (COAD), asthma, hypertension with complications and chronic renal failure were important underlying peril elements of disease severity, non-improvement and case fatality in SARS-COV-2 patients.

\section{LIMITATIONS}

1) Clinical symptoms, laboratory tests (CT chest etc.) and or documentation were incomplete in few patients. Nevertheless, considering the demand to dispense objective data and the compelling agenda, patients were not approached for further biological laboratory sampling or extra history.

2) Our study sample was short because we included only moderately severe COVID-19 patients in our study and patients with mild illness were excluded from this study.

\section{SUGGESTIONS / RECOMMENDATIONS}

This study interprets that early usage of NIV (HFNO, CPAP) diminished respiratory failure symptoms, worsening of disease and the needs for invasive ventilation. During the months of May and June2020 many patients presented with COVID-19 pneumonias in ICU of DHQ Sargodha with moderate to severe respiratory failure and were managed with pharmacological treatments, NIV and mechanical ventilation. Study also concludes that proper careful nursing care, combination of broad-spectrum antibiotics, steroids, prone positioning and nutritional support plays important role in managing moderately severe COVID-19 patients. 


\section{CONFLICT OF INTEREST / DISCLOSURE}

None.

\section{ACKNOWLEDGEMENTS}

Me (Dr. Amar Nazir) especially thankful to Dr. Nazia Nazir \& Muhammad Mustafa Qamar for contributing in this study.

\section{REFERENCES}

1. Zhu N, Zhang D, Wang W, Li X, Yang B, Song J, et al. China Novel Coronavirus Investigating and Research Team. A Novel Coronavirus from Patients with Pneumonia in China, 2019. N Engl J Med. 2020;382(8):727-33.

2. Abid K, Bari1 YA, Younas M, Javaid ST, Imran A. Progress of COVID-19 Epidemic in Pakistan. Asia Pac J Public Health. 2020;32(4):154-6.

3. Chen N, Zhou M, Dong X, et al. Epidemiological and clinical characteristics of 99 cases of 2019 novel coronavirus pneumonia in Wuhan, China: a descriptive study. Lancet. 2020;395(10223):507-13.

4. Ruan Q, Yang K, Wang W, Jiang L, Song J. Clinical predictors of mortality due to COVID-19 based on an analysis of data of 150 patients from Wuhan, China. Intensive Care Med. 2020;46(5):8468.

5. Huang C, Wang Y, Li X, et al. Clinical features of patients infected with 2019 novel coronavirus in Wuhan, China. Lancet 2020;395(10223):497-506.

6. Moore JB, June CH. Cytokine release syndrome in severe COVID19. Science. 2020;368(6490):473-4.

7. Elmy YA, Fawzy M, Elaswad A, Sobieh A, Kenney SP, Shehata AA. The COVID-19 pandemic: a comprehensive review of taxonomy, genetics, epidemiology, diagnosis, treatment, and control. J Clin Med. 2020;9(4):1225.
8. Belouzard S, Millet JK, Licitra BN, Whittaker GR. Mechanisms of coronavirus cell entry mediated by the viral spike protein. Viruses. 2012;4(6):1011-33.

9. Arons MM, Hatfield KM, Reddy SC, et al. Presymptomatic SARSCoV-2 infections and transmission in a skilled nursing facility. $\mathrm{N}$ Engl J Med. 2020;382(22):2081-90.

10. Bai Y, Yao L, Wei T, Tian F, Jin DY, Chen L eta al. Presumed asymptomatic carrier transmission of COVID-19. JAMA. 2020;323(14):1406-7.

11. Balachandar V, Mahalaxmi I, Kaavya J, Vivekanandhan G, Ajithkumar S, Arul N, at al. COVID-19: emerging protective measures. Eur Rev Med Pharmacol Sci. 2020;24(6):3422-5.

12. Feldstein LR, Rose EB, Horwitz SM, Collins JP, Newhams MM, Son $\mathrm{MBF}$, et al. Multisystem Inflammatory Syndrome in U.S. Children and Adolescents. N Engl J Med. 2020;383(4):334-346.

13. Zhang J, Wang X, Jia X, Li J, Hu K, Chen G, et al. Risk factors for disease severity, unimprovement, and mortality in COVID-19 patients in Wuhan, China. Clin Microbiol Infect. 2020;26(6):767-72.

14. Tabata S, Imai K, Kawano S, et al. Clinical characteristics of COVID-19 in 104 people with SARS-CoV-2 infection on the Diamond Princess cruise ship: a retrospective analysis. Lancet Infect Dis. 2020;20(9):1043-50.

15. Wang D, Hu B, Hu C, Zhu F, Liu X, Zhang J, et al. Clinical Characteristics of 138 Hospitalized Patients With 2019 Novel Coronavirus-Infected Pneumonia in Wuhan, China. JAMA. 2020;323(11):1061-9.

16. Guan WJ, Ni ZY, Hu Y, Liang WH, Ou CQ, He JX. Clinical characteristics of coronavirus disease 2019 in China. N Engl J Med. 2020;382(18):1708-20.

17. Mehta P, McAuley DF, Brown M, Sanchez E, Tattersall RS, Manson JJ; HLH Across Speciality Collaboration, UK. COVID-19: consider cytokine storm syndromes and immunosuppression. Lancet. 2020;395(10229):1033-4.

18. Verity R, Okell LC, Dorigatti I, et al. Estimates of the severity of coronavirus disease 2019: a model-based analysis. Lancet Infect Dis. 2020;20(6):669-7. 